\title{
El enfoque pedagógico en la enseñanza de la primera lengua en Uruguay a través de algunas reflexiones didácticas del período 1940-1970
}

Juan Manuel Fustes

Instituto de Educación, Instituto de Lingüística, Centro de Lenguas Extranjeras,

Universidad de la República, Uruguay /

juanmanuelfustes@gmail.com

Trabajo recibido el 30 de marzo de 2018 y aprobado el 28 de mayo de 2018 .

\begin{abstract}
Resumen
En este artículo discutimos el marco para la consideración de una teoría de la enseñanza de la lengua sobre la base de la identificación de un enfoque pedagógico que se ve en ella como producto circunstancial. Con la atención puesta específicamente sobre el Uruguay del período 1940-1970, analizamos varios textos escritos por lingüistas, filólogos, maestros y profesores cuyo objetivo era establecer algunas condiciones prácticas y teóricas para la enseñanza de la primera lengua. En esos textos, encontramos particularmente un elemento pedagógico cuya presencia interfería con la reflexión sobre la naturaleza epistémica de la lengua y sobre la relación entre hablante y lengua.
\end{abstract}

The pedagogical approach in the teaching of the first language in Uruguay through some didactic reflections of the period 1940-1970

\footnotetext{
Abstract

In this paper, we discuss a framework for the consideration of a theory of the language teaching based on the identification of a pedagogical approach that is seen in it as a circumstantial product. With the attention specifically focused on Uruguay during the period 1940-1970, the work analyses several texts written by linguists, philologists, school teachers and professors whose aim was to settle some practical and theoretical conditions to the teaching of the first language. In these texts we found, in particular, a pedagogical element, whose presence interfered with the reflection on the epistemic nature of language and with the relationship between speaker and language.
}

\section{Palabras clave}

teoría de la enseñanza pedagogía

lingüistica y enseñanza de lenguas

\section{Keywords}

theory of teaching pedagogy linguistics language teaching 


\section{O enfoque pedagógico no ensino da primeira língua no Uruguai através de algumas reflexões didáticas do período 1940-1970}

\begin{abstract}
Resumo
Neste artigo discutimos o marco para a consideração de uma teoria do ensino da língua baseado na identificação de um enfoque pedagógico, que é visto nela como um produto circunstancial. Com a atenção posta especificamente no Uruguai do período 1940-1970, analisámos vários textos escritos por linguistas, filólogos, mestres-escolas e professores cujo objetivo era estabelecer algumas condições práticas e teóricas para o ensino da primeira língua. Nesses textos encontrámos, particularmente, um elemento pedagógico cuja presença interferia com a reflexão sobre a natureza epistêmica da língua e sobre a relação entre falante e língua.
\end{abstract}

\section{Presentación general}

Los materiales aportados en este artículo provienen de mi trabajo de tesis denominado "Las concepciones sobre la enseñanza de la lengua en la escena académica uruguaya entre 1920 y 1970" (Fustes 2016) que realicé en la Maestría en Ciencias Humanas de la Universidad de la República ${ }^{1}$ bajo la dirección del Prof. Luis Behares. Esta investigación se llevó a cabo entre los años 2013 y $2016^{2}$ y estuvo signada por un enfoque de las dimensiones de la enseñanza que la hacen depender de su sustento en el lenguaje (siguiendo la óptica de Behares 2007).

Para ese trabajo sopesé, en ocasión de la enseñanza de la lengua ${ }^{3}$, los aspectos teóricos de la lengua como objeto de estudio y del lugar del profesor y del alumno como hablantes.

A partir de uno de los puntos hallados en esa investigación, trataré de pro-

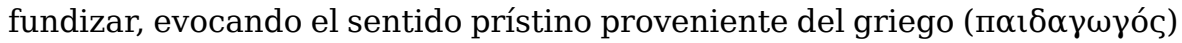
y sus continuidades en la era contemporánea, en lo que llamaré pedagogización de las reflexiones sobre la enseñanza de la lengua. Se trata de observar y dar cuenta de la forma en que el Uruguay fue imprimiendo rasgos pedagógicos sobre sus propuestas de enseñanza de la primera lengua. Esto se observará a través de textos teóricos producidos en el ámbito intelectual y académico uruguayo dentro del período que seleccionamos para esta presentación (1940-1970).

Nos centraremos, por lo tanto, en dos objetivos principales. Por un lado, será importante presentar el marco general en que esta investigación se desarrolló, tanto en los aspectos teóricos como en los históricos. Será de particular interés mostrar cómo se entiende lo pedagógico en la enseñanza desde una lectura de la teoría de la enseñanza atravesada por la noción de sujeto de lenguaje. Por otro lado, nos importará hacer ver algunos ejemplos de análisis dentro de un subconjunto de textos (respecto de los 16 originales, que iban desde 1920 hasta 1970) que abarca 12 documentos publicados entre 1940 y 1970 (que serán definidos por intensión y extensión en el apartado 5).

\section{Palavras-chave}

teoria do ensino pedagogia linguistica ensino de línguas

1. Para la realización y la finalización de esta Maestría conté con el apoyo de la Comisión Académica de Posgrado de la Universidad de la República.

2. Está inserta en el trabajo de la línea Dimensiones Lenguajeras de la Enseñanza y el Aprendizaje (Instituto de Educación, FHCE, UdelaR).

3. Pensando en aquella lengua que debía ser considerada como primera para los alumnos de las escuelas uruguayas. Se nos ocurre rápidamente que esta sea el español pero, en la década de 1960, también aparecerá la discusión sobre el lugar del portugués, tema que no antenderemos en este artículo. 


\section{Antecedentes}

Hemos de considerar como antecedentes a este trabajo aquellos que se adscribirían a un área temática llamada enseñanza escolar de la primera lengua en el Uruguay, especialmente en sus aspectos teóricos, históricos y sociolingüísticos.

Para sus aspectos teóricos, podemos incluir todo lo que se centre en la teoría de la enseñanza de la lengua, las concepciones de saber/conocimiento, lengua y hablante presentes en textos que aspiran a un desarrollo teórico sobre la cuestión de la enseñanza de la primera lengua en el Uruguay. Las cuestiones históricas pueden entrar en la historia de las ideas lingüísticas, la historia de las instituciones escolares y del desarrollo de las actividades científico-académicas en el país. El lado sociolingüístico puede comprender todo lo que enmarque, desde descripciones sociolingüísticas, la relación entre las variedades lingüísticas, los procesos de estandarización y las implicancias político-lingüísticas de la enseñanza de la lengua institucionalizada en el Uruguay.

Tomamos, a efectos de los objetivos de esta indagación, dos grandes grupos de antecedentes: las investigaciones que han trabajado sobre lengua y escuela en el Uruguay del siglo XX (§ 2.1) y las investigaciones que se han dedicado a trabajar teóricamente sobre la enseñanza (§ 2.2).

\subsection{En lengua y escuela en el Uruguay del siglo XX}

En un sentido histórico general, podemos considerar las investigaciones que nacieron en la década de 1990 acerca de la oralidad y la escritura en los primeros tiempos del asentamiento europeo en la margen oriental del Río de la Plata: allí tenemos textos como la compilación de Behares y Brovetto (1994) o el artículo de Coll (1994).

El interés desde una lectura sociolingüística de los aspectos políticos, ideológicos implicados en los procesos de estandarización y de los hitos legales e institucionales en el Uruguay, generó estudios como los de Barrios et al. (1993) y Barrios $(2009,2011)$. Asimismo, podemos señalar los trabajos de Oroño (2010, 2011, 2014, 2016a, 2016b), que nos sitúan en el marco histórico y temático que nos interesa, desde visiones de las representaciones e ideologías lingüísticas en el Uruguay de los siglos XIX y XX hasta estudios sobre el papel de los manuales escolares en la enseñanza de la lengua española en el Uruguay.

\subsection{En teoría de la enseñanza}

Es de particular relevancia el trabajo realizado, principalmente en la última década, a partir del reconocimiento de un campo teórico de la enseñanza, reflejado en producciones de las líneas Estudio de lo didáctico como acontecimiento discursivo e intersubjetividad (2002-2008) e Indagaciones en el campo teórico de la enseñanza (2009-2015), las cuales se desarrollaron en la Universidad de la República (Facultad de Humanidades y Ciencias de la Educación). En los trabajos de esas líneas (Behares, 2005, 2007, 2008, 2011; Bordoli 2011; Fernández 2006, 2007, 2008; 2010; Fustes 2010, 2014; Venturini 2012; entre otros) se transitó principalmente por la pregunta sobre la enseñanza ligada al sujeto del lenguaje, en diálogo con la teoría del sujeto propia de las elaboraciones de J. Lacan ${ }^{4}$, mas también con refe-

4. Por ejemplo, a partir de la senda abierta en el texto "Función y campo de la palabra y del lenguaje en psicoanálisis" (Lacan 1966). 
rencia a los planteos sobre la lengua y el signo que pueden hallarse en el Curso de lingüística general de F. de Saussure (1916). Se atendió también a la revisión de la lingüística en sus aspectos epistemológicos y en términos de sujeto-hablante impulsada por Jean Claude Milner, entre varios textos, en Introducción a una ciencia del lenguaje (Milner 1989) y El amor por la lengua (Milner 1978).

Estimamos que estas indagaciones son antecedentes a nuestro trabajo, en tanto la investigación trató de dejar en evidencia algunos aspectos particulares de la enseñanza de la lengua que no habían sido tratados hasta el momento en ese marco.

\section{Algunas precisiones teóricas}

La investigación se posicionó respecto de las nociones de hablante y enseñanza, en tanto se tomó en cuenta la lengua como saber/conocimiento y las dificultades de su representación para ser enseñado. A efectos de pensar así la lengua, es ineludible la referencia al desarrollo teórico generado en Milner (1978), dado que ese texto plantea una epistemología para la lingüística en su afectación por las nociones psicoanalíticas lacanianas de sujeto y de lenguaje.

Cuando se trata de pensar en la enseñanza de la lengua, y una vez que nos preguntamos sobre la naturaleza epistémica de ese objeto de enseñanza, lo discutido en el marco de la lingüística resulta pertinente, más allá de que no siempre ese marco de ciencia moderna requiera o admita una discusión sobre la propia naturaleza del objeto que estudia. Podemos señalar allí una distancia pertinente, a los efectos teóricos que nos interesan, entre las lenguas y la lengua. Por un lado, la lingüística se remite a las lenguas tradicionalmente establecidas (español, francés, inglés, etc.), pero, por otro lado, también puede considerar la lengua como lo simbólico, el modelo de los sistemas semióticos o del funcionamiento social, cultural y conceptual humano, una estructura supraestante que define al hablante-sujeto ${ }^{5}$.

Así como la lengua es asunto pertinente para nuestra lectura, el signo lingüístico también es colocado como requisito para el estudio de la convergencia entre enseñanza, hablante y saber/conocimiento de lengua. La teoría del signo permite un rastreo a lo largo de la historia que puede conocer capítulos antiguos en Crisipo de Solos (2008), medievales en San Agustín $(1873)^{6}$, pero alcanza también en F. de Saussure una síntesis y una proyección hacia los tiempos recientes.

Las lecturas de la teoría saussuriana del signo se presentan en una inevitable variedad (conforme se revisa, por ejemplo, en Pardo 2011, Suenaga 1999 o Melo y Carvalho 2014), lo cual (además de la multiplicidad de las interpretaciones que el lenguaje suscita) se puede deber a las oscilaciones presentadas en el mismo Cours de linguistique générale. Por momentos, en el signo lingüístico, significado y significante parecen verse en forma correspondiente y simétrica (sobre todo si nos atenemos a los famosos esquemas ovales); sin embargo, los aspectos de mutabilidad, inmutabilidad y arbitrariedad, sumados a las determinaciones mostradas en las relaciones sintagmáticas y paradigmáticas, muestran la prevalencia de un orden del lenguaje, que se impone al hablante ${ }^{7}$ y lo define en tanto tal ${ }^{8}$.
5. Tratamos esta cuestión principalmente en ocasión del apartado del Cours de Linguistique Générale que lleva el título de "Place de la langue dans les faits de langage" (Saussure 1916, 27-32) en Fustes (2010).

6. Ya mencionado o discutido por Eco (1973), Todorov (1977) o, con más especificidad, en Behares (2008, 2010).

7. Algunas menciones coincidentes al sustento saussuriano de la determinación del orden de la lengua sobre el hablante pueden ser leídas en Henry (1992).

8. La revisión de la psicolingüística (como disciplina en auge en el medio estadounidense entre las décadas de 1960 y 1980) propuesta en Brasil por Cláudia Lemos (1995, 2000, entre otros) también siguió la dirección de retorno a un sujeto no psicológico (de procesos mentales) sino constituido por el lenguaje o efecto de este. La emergencia de esta lingüística que se propone la explicación del hablante por una vía lingüística (lenguajera deberíamos decir, calcando el francés langagière) significó parte importante de la influencia teórica que llevó a las producciones sobre sujeto, lenguaje y enseñanza (Behares et al. 2004). 
El hablante se halla en el juego de una lengua previamente dada, cuya materialidad es inmotivada. Él no puede decidir cambiarla, pero puede, sin darse cuenta, toparse con un signo nunca acontecido antes. El hablante opera en la medida en que toma eso que es previamente dado, que está colocado del lado del Otro, es decir, de la otredad que es el mundo humano en el que el hablante adviene.

Ese material que se impone, los sonidos lingüísticos que nos envuelven, en su faceta más real, aun antes de nuestro nacimiento, componen una sustancia que se ordena de un modo particular, en función de su aspecto significante. Visto así, el signo queda desimetrizado y el significado queda reservado para la sutura final, el momento de la interpretación particular y única acontecida en cada hablante y en cada instante. Estamos leyendo la cuestión del signo aquí también a la luz de los órdenes Real, Simbólico e Imaginario teorizados en el psicoanálisis lacaniano, enviados luego a la lingüística para incidir sobre sus elementos fundacionales ${ }^{9}$.

Al colocarnos del lado de la búsqueda permanente de viabilidad, de estabilidad, la ilusión de representar el mundo mediante el lenguaje, vemos en los movimientos de etiquetar, describir, ordenar y, en una especial instancia, al explicar a las nuevas generaciones o simplemente a quienes pretenden saber. Nos hemos adentrado, casi sin darnos cuenta, en lo que es del orden de la enseñanza.

El acto de enseñar posee todos los elementos puestos en juego: el saber, la representación, el lenguaje, la transmisión, la transferencia, las suposiciones de saber del otro, el deseo de saber, el goce de la enunciación, los movimientos relacionales en que lo erótico entra en juego (Behares 2007). En ese camino estamos viendo una teoría de la enseñanza como derivada de una teoría del lenguaje, inmersa en la cuestión del sujeto-hablante. Más básicamente, y en general, estamos proponiendo la posibilidad de rastrear una teoría de la enseñanza a lo largo de diferentes lugares y épocas. Behares $(2007,2)$ nos plantea las dificultades para hallar una teoría de la enseñanza en cuanto a que

no es obvio que se deba admitir la existencia de una teoría de ese tipo, aunque, sin dudas, podría parecer imposible su ausencia, ya que su necesidad está sugerida por la comprobación universal de prácticas llamadas 'enseñanza' y por la presencia de textos que se refieren a ellas (Behares 2007, 2).

La teoría de la enseñanza es un campo esporádicamente reconocido en las tradiciones occidentales, muchas veces difuso y perdido entre los tratamientos filosóficos sobre el saber/conocimiento y el lenguaje, o incluso presente en los estudios sobre los contextos institucionales en que la enseñanza ha sido visualizada como existente. Así, la gama de posibilidades recorre desde su ligazón con el lenguaje y el saber/conocimiento hasta sus frecuentes asociaciones y convivencias con lo pedagógico, aquello que es del orden del acompañamiento al crecimiento de los niños. De hecho, si nos atenemos a lo producido desde las últimas décadas del siglo XIX, notamos la prevalencia de la enseñanza pedagogizada, colocada en el marco de la educación, con miras al desarrollo y al crecimiento de los niños, y también en estrecha relación con el aprendizaje, enfocado este desde la psicología, lo que abre posibilidades de medición de la eficacia de la enseñanza como acción tendiente a modificar pensamientos y conductas ${ }^{10}$. Esto da como resultado una teoría de la enseñanza pensada dentro de los marcos
9. Si nos propusiésemos tomar los términos teóricos en su devenir, deberíamos más bien reconocer que Lacan se nutre de la lingüística para realizar sus formulaciones, y son luego lingüistas como Jean-Claude Milner quienes admiten los efectos sobre la lingüística de las propuestas lacanianas. Las formulaciones lacanianas al respecto fueron presentadas por primera vez en el año 1953 (Lacan 1982) y profundizadas a lo largo de más de veinte años, especialmente en su Seminario 22 (Lacan 1974-75). Una parte de la filosofía europea contemporánea fue afectada por estas perspectivas (piénsese en nombres como A. Badiou o S. Žižek).

10. Behares (2005, 2007) y Fernández (2006) han subrayado la coalición entre enseñanza y educación operada desde la apertura de la Modernidad, marcando en el siglo XVII su hito con la Didáctica magna de Comenio (Comenius 1986), que significa proponer una enseñanza "naturaleza instrumental (tecnológica) y en una relación de transparencia entre los sujetos" (Fernández 2006, 25), lo cual se traduce en un "interés psicológico y moral por el niño" orientado a "convertir a los niños en hombres razonables y adaptados a la cultura" (Fernández 2006, 25). 
educativo (de formación en base a un proyecto social y político pensado para las nuevas generaciones) y pedagógico (de acompañamiento al desarrollo de los niños en su transformación a la adultez).

En ese contraste entre lo propio de la enseñanza (lenguaje, sujeto, saber/ conocimiento) y aquello donde la enseñanza participa (aprendizaje, orden educativo, pedagógico) vemos el punto de quiebre que nos indicará el elemento llamativo en la lectura de los documentos que estamos por presentar.

\section{Sobre el marco histórico}

Si bien la investigación no se propuso esclarecer aspectos históricos ni aportar nuevos datos, es posible encontrar contribuciones para una lectura que reconstruya aspectos de la historia de la enseñanza del español en el Uruguay, así como de los cruces entre escolarización y enseñanza de la(s) lengua(s) o de la historia de las ideas lingüísticas en el Uruguay. Mencionaremos brevemente algunos aspectos que hacen al marco histórico en que surgieron los documentos seleccionados y analizados en la investigación.

Con el pasar del siglo XIX, el Uruguay se transformó en cuanto a sus posibilidades institucionales para la educación. Desde una educación a cargo de órdenes religiosas ${ }^{11}$ llega a un sistema de enseñanza de nivel primario universalizado, que tiene una continuidad hacia el nivel secundario-universitario. Fueron importantes, para la inspiración filosófica de este ciclo de formación educativa a cargo del Estado, los valores laicos recogidos de los adoptados en Francia o Estados Unidos, a partir de las ideas iluministas.

Aun con escuelas públicas primarias y secundarias esparcidas por todo el país hacia principios del siglo XX, debemos llegar a la década de 1930 para que se visualice al nivel secundario más como una continuación del primario y no tanto como la preparación para la formación universitaria. La escisión de la enseñanza secundaria del ámbito de la Universidad (1935) es un hecho significativo en esa dirección.

En lo que tiene que ver con el lenguaje y las lenguas, su estudio, su investigación y su enseñanza, son capítulos dignos de mención los que implican el desarrollo de las investigaciones filológicas y lingüísticas que tuvieron a Benigno Ferrario ${ }^{12}$, Adolfo Berro García ${ }^{13}$ y Eugenio Coseriu ${ }^{14}$ como figuras destacadas en la primera mitad del siglo XX. Cada una de ellas se asocia, en la época que le corresponde, con una institución diferente.

La alusión a esos tres personajes nos permitió mencionar distintas instituciones en que ocurrió la enseñanza y la investigación sobre lenguaje y lenguas en Uruguay. En el sistema escolar dirigido por el Estado el español fue la única lengua de enseñanza, solamente con pequeños espacios reservados en el nivel secundario principalmente para inglés, francés e italiano, como lenguas extranjeras. La investigación, mezclada algunas veces con la formación profesional, se desarrolló en varias instituciones: en la formación magisterial (consolidada desde 1882 aproximadamente) y en la profesoral (a través del Instituto de Profesores Artigas, desde 1951); el Instituto de Estudios Superiores (1929-1969), la Academia Nacional de Letras (desde
11. Por falta de organización y recursos económicos, el Estado uruguayo fue incapaz hasta cerca de 1880 de poner en marcha y conducir seriamente un sistema institucionalizado de educación y enseñanza.

12. Benigno Ferrario (1887-1959) era un lingüista italiano, nacido en Milán y formado en el Real Istituto delle Lingue Orientali (Nápoles), de donde egresó como filólogo y orientalista (Araújo 1920, 171-172). Llegado a Montevideo en marzo de 1911, se ofreció para dictar cursos en la Universidad y mantuvo su enseñanza en la Facultad de Derecho y Ciencias Sociales hasta el final de su carrera, con la Cátedra de Lenguaje. En las décadas siguientes enseñó paralelamente en el Instituto de Estudios Superiores (Cátedra de Lingüística) y en la Facultad de Humanidades y Ciencias (Cátedra de Lenguas Amerindias).

13. Adolfo Berro García (1885-1969) era abogado de formación, graduado en 1908 y empezó a destacarse en la segunda década del siglo XX como docente de nivel secundario. En 1929 fue fundador del hoy extinto Instituto de Estudios Superiores, donde, entre 1929 y 1943, dictó cursos de especialización en "Lingüística general y comparada", "Gramática histórica de la lengua española”, "Lexicología” y “Fonética” (Berro García 1952, 4) y desde 1934 fue nombrado director de la Sección de Filología y Fonética Experimental de ese instituto. Cuando llegó a ocupar la cátedra de Ciencias del Lenguaje en la Facultad de Humanidades y Ciencias (fundada en 1946), Berro se había dedicado, entre otras cosas, a la producción materiales didácticos para enseñanza de la lengua española (como los que analiza Oroño 2011, 2014) y había participado con posiciones acerca de temas educativos y temas del lenguaje, sobre todo en lo atinente al léxico.

14. Eugenio Coseriu (1921-2002) (Eugen Co eriu) nació en el territorio de la actual República de Moldavia (Mih ileni, Ri cani), en aquel tiempo Rumania. Como lingüista se formó entre la i (Rumania) e Italia (desde 1940). Causas varias, entre las que se contarán seguramente las personales, económicas, políticas y políticouniversitarias lo llevaron al Uruguay, donde ocupó desde 1951 la cátedra de Teoría del Lenguaje y Lingüística General en la nueva Facultad de Humanidades y Ciencias. También es recordada su docencia en la formación de profesores. Co eriu se volvió una figura en condiciones de eclipsar a quienes se habían acercado a los temas del lenguaje en el ámbito local, y aportó para la generación un corte teórico neto con las tradiciones locales, pues sus producciones teóricas realizadas en Montevideo plasman las discusiones de la lingüística moderna que se estaban desarrollando en Europa, principalmente entre Francia, Suiza, Alemania, Italia y Checoslovaquia. Entre 1961 y 1963 concreta su regreso a Europa y se asienta en la Universidad de Tübingen, donde permanecerá hasta el final de su carrera. 
1943) y la Facultad de Humanidades y Ciencias (desde 1946). Todas estas instituciones tienen variadísimos caracteres e historias que no podremos detallar aquí, pero representan también determinadas formae mentis que sí intentaremos delinear más en lo siguiente.

Esas formae mentis, o diferentes tradiciones, ideologías, mentalidades fueron consideradas en nuestra investigación como conformaciones y denominadas como configuraciones discursivas. Llegamos a reconocer y enumerar varias de esas configuraciones y las tomamos como formas de funcionar discursivamente que constituyen la lengua como conocimiento y su enseñanza. Estas configuraciones discursivas están asociadas a figuras personales e instituciones pero también a diferentes grados de compromiso con lo local, lo nacional, lo hispánico, lo educativo, lo pedagógico y lo científico: podríamos nombrarlas como 1) el peculiarismo lingüístico; 2) la filología hispánica; 3) el profesorado de Idioma Español; 4) el magisterio y 5) la lingüística.

La primera de ellas se ve materializada en los estudios llamados costumbristas, folklóricos, en Uruguay realizados por aficionados a temas antropológicos y lingüísticos. Pretenden marcar las particularidades locales, contrastando con el reconocimiento dado al gran discurso de la filología hispánica (que demostraba el peso cultural de lo europeo), de modo de ir conformando un discurso sobre la lengua y la cultura locales. En lo lingüístico, esto se desarrolla principalmente en el estudio del léxico y del elemento indígena superviviente. Figuras que realizaron trabajos de este tipo fueron: José Henriques Figueira, los primeros lexicógrafos Daniel Granada, Washington Pedro Bermúdez y Sergio Washington Bermúdez (antes de 1920), pero también, más adelante, Adolfo Berro García.

La segunda y la tercera conformación están muy emparentadas. Filología fue el nombre que recibió el estudio del lenguaje en el Instituto de Estudios Superiores (y su revista, el Boletín de Filología), uno de los primeros espacios institucionales dedicados a esta temática, lo cual permitía reunir los intereses gramaticales con los literarios y mantener los nexos con las prácticas españolas al respecto. Pero junto con esta institución y la generación que estuvo al frente de ella, esta configuración discursiva se fue diluyendo hacia la década de 1950 para nutrir otras: principalmente la del profesorado de Idioma Español. Algunas figuras pueden ser recordadas a este respecto: Sixto Perea y Alonso, Adolfo Berro García o Luis Juan Piccardo.

La configuración propia del profesorado se desarrolla en un espacio curricular propio de la enseñanza secundaria uruguaya: los cursos de Idioma Español. A partir de lo que pudimos recoger en los documentos, pudimos ver que esa configuración discursiva es fiel a una concepción de lengua española que trasciende lo local y que es prescindente de la dimensión nacional de la lengua. De este manera, no contrasta con la vieja gramática, en tanto da importancia a la prescripción o el modelo que esta presenta, sin dejar de proponerse estudiar, a través de la lengua, el lenguaje y los fundamentos del pensamiento humano.

Es constante en esta conformación, asimismo, el recurso a algún tipo de conocimiento de la lengua proveniente de un corpus externo: la gramática o la lingüística. La pedagogía nutrida por elementos psicológicos también está presente en esta discursividad, lo cual se ve reflejado en el uso del término adolescente para referirse al alumno, como veremos en el apartado 6 . 
La configuración discursiva del magisterio, por su parte, abreva de sus antecedentes magisteriales, se reconoce a partir de la expansión de la escuela pública a un alcance nacional universal, a fines del siglo XIX. La presencia de la psicología en esta configuración es determinante, así como la orientación pedagógica marcada por las influencias de la llamada Escuela Nueva, movimiento que resulta la confluencia de varias iniciativas más o menos teorizadas de educación alternativa a las tradiciones que se venía dando en Europa y Estados Unidos desde fines del siglo XIX ${ }^{15}$.

La discursividad del magisterio está marcada también por los aspectos nacionales y morales propios de una visión que apunta a conformar ciudadanos nuevos, útiles al Estado y a las demás instituciones sociales. Veremos que, al lidiar con el problema de la enseñanza, y por obedecer a fines más altos, la cuestión del conocimiento de la lengua queda en una incómoda posición por efecto del centramiento en el niño, en su aprendizaje y por la preocupación en la intervención sobre él. La única persistencia del conocimiento se da a través de los resabios del modelo anterior (no centrado en el niño): se apela aún a la lengua correcta, la lengua española, el diccionario o la ortografía.

Podemos separar una última atención para la configuración discursiva que llamaríamos de la lingüística. La vemos como definida por la captura del lenguaje y las lenguas como objeto de estudio coherente con la orientación de la ciencia moderna. Esto equivale a decir que defiende el cuidado de la empiria, la equiparación de los objetos de estudio (todas las lenguas, sin importar su prestigio, son dignas de ser estudiadas científicamente ${ }^{16}$ ) y la eliminación de elementos valorativos sobre ellos.

Tiene sus primeros testimonios de presencia en Uruguay con la discusión de las lenguas indígenas entablada por Benigno Ferrario, con afectaciones científicas modernas, dentro de lo que el mismo Ferrario denominaba (por tradición italiana) como glotología.

La configuración discursiva que llamamos filológica tuvo intereses de fundirse y confundirse con esta (como muestra Perea y Alonso 1936), probablemente dado el mayor prestigio que tenían los estudios lingüísticos respecto a los filológicos, que parecían quedarse en el comentario literario culto. Pero la jerarquización de la literatura sobre otras manifestaciones lingüísticas impedía a esos estudios filológicos confundirse con los lingüísticos.

La influencia del discurso que Ferrario ponía de manifiesto fue débil durante varias décadas, hasta que en 1951 Eugenio Coseriu funda su línea de estudios lingüísticos en Uruguay y extiende una conexión, sin pensar en la continuidad con lo que había ni demostrar interés por los objetos de estudio ya frecuentados, sobre todo con la forma mentis de la lingüística que ya estaba presente en Ferrario. La impronta coseriana, en cambio, instala el estructuralismo en pleno debate, con la revisión de Saussure y sus dicotomías (Coseriu 1952, 1958). Hacia la década de 1960, José Pedro Rona es continuador de este enfoque científico, con un cariz sociodialectológico que recibe influencias anglosajonas norteamericanas.

\section{Aspectos metodológicos}

Para llegar a un conjunto limitado de documentos de análisis, hemos tomado aquellos que intentan abordar cuestiones propias de las relaciones entre
15. Aquello que hace posible que se las considere un solo movimiento de pedagogía progresista es la ruptura dada a través del ingreso de un nuevo concepto de alumno, mucho más posible de asociar con un sujeto de aprendizaje afín a la psicología positivista (Venturini 2015). Podemos mencionar como figuras de referencia, llegando más temprano o más tarde a aportar influencias de distintas maneras, a Ovide Decroly (Bélgica, 1871-1932), Célestin Freinet (Francia, 1896-1966), María Montessori (Italia, 1870-1952), o John Dewey (Estados Unidos, 1859-1952).

16. Recuérdese el famoso fragmento del Cours de linguistique générale que reza: "la matière de la linguistique est constituée d'abord par toutes les manifestations du langage humain, qu'il s'agisse des peuples sauvages ou des nations civilisées, des époques archaïques, classiques ou de décadence, en tenant compte, dans chaque période, non seulement du langage correct et du 'beau langage', mais de toutes les formes d'expression" (Saussure 1916, 20). 
lenguaje y enseñanza o las pertenecientes a la enseñanza de la lengua, lo que se traduce en artículos y libros, publicados por diferentes medios de difusión académica, que se proponían tratar estos aspectos teóricos esenciales y cuyos lectores eran generalmente maestros y profesores.

El conjunto generado consiguió un cierto equilibrio, con autores uruguayos y otros no nacidos en el país, pero que desarrollaron en el Uruguay la mayor parte de su vida formativa o profesional. Algunos de los textos fueron leídos masivamente en alguna época, pero otros quedaron confinados a un olvidado estante de la Biblioteca Nacional. Sin embargo, este no ha sido criterio determinante para la inclusión dentro del conjunto destinado al análisis.

Ellos se constituyen en conjunto a efectos de este análisis, no conforman un intercambio de lecturas, oposiciones ni debate. Aun con todas las diferencias que hay entre ellos, pueden ser tomados como aportes individuales pero reunibles en un conjunto.

Para la periodización que proponemos en este artículo (que es una subperiodización respecto del trabajo de tesis), tomamos en cuenta doce textos, publicados entre 1940 y 1970, presentados sinópticamente en el cuadro 1.

\begin{tabular}{|c|c|c|}
\hline Año & Autores & Título \\
\hline 1940 & José Pereira Rodríguez & $\begin{array}{l}\text { Enseñanza de la redacción y la composición: } \\
\text { teoría y práctica }\end{array}$ \\
\hline 1941 & $\begin{array}{l}\text { A. Berro García, C. Martínez } \\
\text { Vigil, V. Pérez Petit, A. Rusconi, } \\
\text { J. G. Antuña, J. del Rey, E. } \\
\text { Laferrière yJ. Pereira Rodríguez }\end{array}$ & $\begin{array}{l}\text { La enseñanza del Español en los Liceos de la } \\
\text { República }\end{array}$ \\
\hline 1943 & Américo Mibelli & $\begin{array}{l}\text { Lenguaje, palabra, gesto en la enseñanza } \\
\text { media uruguaya }\end{array}$ \\
\hline 1949 & $\begin{array}{l}\text { Andrés Da Silva y María Elena } \\
\text { Guzmán de Da Silva }\end{array}$ & Poemario metodológico de la lengua española \\
\hline 1955 & Jesualdo Sosa & Metodología de la expresión infantil \\
\hline 1956 & Luis Juan Piccardo & Gramática y enseñanza \\
\hline 1956 & Alberto Rusconi & $\begin{array}{l}\text { Metodología de la enseñanza de la lengua } \\
\text { española y la literatura en secundaria }\end{array}$ \\
\hline 1958 & Héctor Enrique Solari & Hablar, leer y escribir \\
\hline 1959 & Floro Olano Pagola & Adquisición del signo \\
\hline 1963 & José Pedro Rona & $\begin{array}{l}\text { Relación entre la investigación dialectológica y } \\
\text { la enseñanza de la lengua materna }\end{array}$ \\
\hline 1964 & Celia Mieres & Cursos de Idioma Español en los Liceos \\
\hline 1969 & Héctor Balsas & El maestro frente a la enseñanza de la lengua \\
\hline
\end{tabular}

Cuadro 1: Documentos tomados en cuenta en este análisis (sus referencias completas se encuentran en la sección de bibliografía).

\section{En los textos: enseñanza, saber/conocimiento y hablante-alumno}

Trataremos de dar cuenta de los rasgos de pedagogización reflejados en la enseñanza de la primera lengua en Uruguay a través dos aspectos teóricos: 1) la construcción teórica de un alumno como sujeto pedagógico (§ 6.1);2) 
el tratamiento de la lengua como saber/conocimiento ante la enseñanza, es decir, la cuestión epistémica inherente a toda enseñanza (§ 6.2). En este caso, es determinante la contextualización de la enseñanza de la lengua en la escuela (primaria y secundaria), por lo tanto con el sentido educativo y pedagógico que venimos delineando a lo largo de este texto.

\subsection{El sujeto pedagógico}

Los horizontes pedagógicos establecidos dentro del marco de nuestros textos exigen la existencia de una meta de las acciones pedagógicas. La figura del alumno surge y se define en función de ese punto de vista. Esa figura del alumno como objeto de las atenciones pedagógicas es lo que denominamos sujeto pedagógico.

Los rasgos que constituyen a este sujeto son esencialmente:

1) sus carencias respecto a un modelo deseado por la escuela, es decir, el contraste que surge entre los niños que asisten a las aulas (al menos la percepción que se tiene de ellos) y los que la escuela desea producir;

2) su carácter de sujeto en desarrollo, que debe ser tomado por los cuidados pedagógicos, que serán oportunos en función de su edad evolutiva.

Tanto se coloque el énfasis sobre una u otra forma de capturar al sujeto pedagógicamente, veremos que, en general, la elaboración de esta figura está determinada por datos sociales o culturales, psicológicos y biológicos, los cuales dan con claridad las faltas que la intervención pedagógica estaba llamada a colmar.

De este modo, veremos, sin perder la mira en que nuestros textos versan sobre la enseñanza de la lengua, que en ellos se marcan el contraste cultural, los fundamentos psicológicos del aprendizaje y de la personalidad, el rol pedagógico del maestro y el foco colocado sobre el aprendizaje.

Podemos ver en el texto de Américo Mibelli (1943) retratada la realidad cultural en la que, según el autor, vivían muchos alumnos de las escuelas secundarias uruguayas (tal vez montevideanas) que poblaban las aulas en la década de 1940. Hace mención a lo vulgar y lo distinguido como formatos o tendencias culturalmente opuestas, pero su planteo no recae directamente en los grupos sociales concretos. La discusión de Mibelli se mantiene en el plano del contraste entre dos modelos: lo irracional, lo efímero, lo intrascendente, contra lo racional, lo perdurable y lo decisivo que propone el mundo de la lengua escrita y el pensamiento. La acción pedagógica vendría a operar, entonces, a favor del abandono de un cierto tipo de cultura vulgar para el encuentro de otra racionalidad relativa al mundo de la lengua escrita, para lo que la enseñanza de la lengua se estima fundamental.

Mibelli opone, al modelo que la acción pedagógica debe tener presente, lo que llama "la retórica del 'conventillo'", la cual incluye un desprecio por "las flores de la cultura, que les saben a invernadero" (Mibelli 1943, 14). De allí que se pregunte "¿por qué se trata de los que no estudian, en una metodología para la enseñanza? [...] Porque los frenos con que se nos traba proceden de la existencia de una clase inculta y desvalida dominante numérica en la sociedad" (Mibelli 1943, 15, cursivas del autor). Encuentra 
incluso un trasfondo social y político para leer esta situación: "existen todavía multitud de fuerzas que pugnan contra la extensión de la cultura porque medran con la ignorancia" (Mibelli 1943, 18).

El punto de vista pedagógico recurre también a las perspectivas biológicas y psicológicas. Se otorga, de este modo, un espacio de confianza a las informaciones proporcionadas por las ciencias modernas, entre las cuales la psicología llega con su enfoque del sujeto como máquina de procesos internos y externos, de conductas sustentadas en configuraciones mentales o cerebrales, que también, por ende, obedecen a bases biológicas. Así, por ejemplo, Da Silva y Guzmán mencionan aspectos fisiológicos de los procesos mentales: hablan del alumno diciendo "cuando la obra literaria [...] cobre vida en su cerebro..." (Da Silva y Guzmán 1949, 41) o "el trozo literario que habita en el núcleo de su masa encefálica" (Da Silva y Guzmán 1949, 42), y describen que en el aprendizaje de nuevas palabras en estos términos:

la resonancia afectiva de su significado impresionará la (p)siquis (sic) del adolescente, de tal manera, que unido a ello tendrá retenidos en su representación mental la acentuación y los fonogramas de la palabra aprehendida (Da Silva y Guzmán 1949, 59).

Mieres también transita por un enfoque psicológico cuando dice que el lenguaje
[...] se adquiere, predominantemente, por imitación; el individuo está impulsado en este aprendizaje por la misma fuerza vital: necesidades motoras, actividades volitivas, lúdi- cas; tendencias de dominio (algunas próximas a la magia); búsqueda de satisfacciones que proporcionan la audición y el ejercicio muscular, etc. Imitación, juego, maduración (Mieres 1964, 88).

Se ve en ese pasaje un interés en el seguimiento psicológico de la maduración, la evolución o - dicho en los términos que luego serán más frecuentes- el desarrollo.

Este foco colocado a la vez en el aprendizaje y en el desarrollo (con sus etapas evolutivas, consideradas y nombradas por la mayoría de nuestros autores) concede un lugar especial, siempre que el caso lo requiera, al término adolescente con, al menos, una incipiente elaboración conceptual de la adolescencia. Así, Mibelli, sin utilizar directamente ese término, nos habla del alumno diciendo que "su vida interior evoluciona desde el ciclo lúdico hacia el onírico. Segunda etapa del desarrollo cerebral" (Mibelli 1943, 18), a lo que se agrega el elemento sexual como rasgo biológico, "sexualidad incipiente y, con frecuencia, imprecisa" (Mibelli 1943, 18).

Da Silva y Guzmán muestran claramente el rol pedagógico del maestro o profesor ante su alumno adolescente: "el profesor tiene que ser indudablemente, el representante de la forma espiritual más acabada, en la hermenéutica de la adolescencia" (Da Silva y Guzmán 1949, 29). Este punto de vista está presente en más textos, aunque con diferentes matices. En Pereira Rodríguez, el adolescente será objeto de educación y de instrucción con el fin de aportar a una "mente en formación" (Pereira Rodríguez 1940, 45). También Rusconi visualiza al adolescente, quien está "deseoso por descubrir, discutir y conversar para participar en situaciones que reputa importantes para revelar su personalidad" (Rusconi 1956, 12). 
Vemos también en Olano (1959) y Balsas (1969) la propuesta de una enseñanza adecuada a las etapas evolutivas. Olano afirma la importancia de "la ordenación, el ajuste a los nuevos conocimientos y la consonancia con el medio y con la edad mental del adolescente" (Olano 1959, 107). Balsas establece: "estamos de acuerdo con quienes piden moderación, cercenamiento de temas o aplicación de enfoques acordes con la psicología del alumno" (Balsas 1969, 27-28).

Otra faceta de la influencia de la psicología en la conformación de este alumno como sujeto pedagógico es la proveniente de las formulaciones teóricas sobre la personalidad. Este enfoque permitió clasificar con adjetivos a los alumnos según su forma de comportarse o relacionarse con el mundo, lo cual daba al profesor o maestro un cuadro de previsibilidad. Menciones al término personalidad, que podrían evocar este punto de vista, pueden ser observadas en Da Silva y Guzmán (1949), Sosa (1955), Rusconi (1956), Solari (1958), Olano (1959) y Mieres (1964). Sin embargo, no todos los textos coinciden en ese concepto, pues remiten a él con pocas definiciones y terminan dándolo por descontado, como si proviniese de un cierto sentido común ${ }^{17}$. Se lo resalta más bien con el objetivo de defender el tratamiento de la singularidad, aquello que no ha de someterse a reforma sino, más bien al contrario, que puede ser reafirmado gracias a la labor pedagógica, la cual, por ejemplo, para Mieres, se dirige al "desarrollo integral de la personalidad del educando" (Mieres 1964, 93). La personalidad puede ser, además de un punto a tener en cuenta entre lo pedagógico, un factor considerado al pensar en la enseñanza, como nos indica Rusconi:

no pretenda el profesor reducir su enseñanza de la composición a un común denominador. Hay alumnos introvertidos y extravertidos, atentos y distraídos, serios y humoristas, reflexivos y atropellados, apagados y vivaces. Es menester tomar en consideración estas diferencias (Rusconi 1956, 9).

En la investigación realizada hemos indicado (Fustes 2016, 147-168) también una serie de atribuciones hechas sobre el alumno, que tocan los caracteres político, psicológico, sobre la relación lenguaje-pensamiento, epistémicas, sobre el hablante de una lengua, sociológicas, morales y de la relación maestro alumno como relación pedagógica, así como hemos encontrado posibles lecturas en una dirección psicoanalítico-discursiva. Algunas de estas llamadas atribuciones convergen para construir ese sujeto pedagógico, en tanto surge del cruce de concepciones psicológicas (de diversa índole: aprendizaje, personalidad, desarrollo), sociológicas, políticas y morales. Sin embargo, hay un especial apartado que se puede dedicar a lo que se coloca sobre el alumno como hablante. Los elementos implicados allí son de diversa índole, pues pueden verse los aspectos lingüísticos, su relación con determinadas lenguas reconocidas y posibles de enseñar (español, portugués, inglés, etcétera) pero también los aspectos abarcadores de lo lenguajero, es decir, más ligados a la singularidad de la lengua materna, que rehúye de posibles nombramientos y consistencias (sin perder de mira el enfoque de Milner (1978)).

Nos concentraremos, en el apartado siguiente, en el punto crucial que permite pensar en la enseñanza, es decir, la forma en que se pone en juego la lengua en tanto que saber/conocimiento. Prestaremos especial atención, para esta ocasión a lo que vemos como efectos del enfoque pedagógico sobre la concepción epistémica.
17. Esta teoría de la personalidad que se sigue en algunos escritos carece de un fuerte marco de referencia identificado con obras o autores. En Da Silva y Guzmán (1949) se presenta un cuadro sinóptico de la psicología del alumno, que hace referencia a necesidades (menesteres), intereses (anatocismo), incitantes y angustias, con alusión al concepto de personalidad colocado en relación con la producción escrita. Para esto, se recurre a la referencia de Constantin Muresanu (1930) autor que llega a ellos probablemente por la cita que de él hace Edouard Claparède en su obra publicada en español como La psicología y la nueva educación (Claparède 1933). 


\subsection{La renuncia y el relegamiento epistémicos}

Al reconocer la cuestión epistémica como la ligada a la constitución del objeto de enseñanza y al procurar leer en los textos el planteo de ese problema, encontramos menciones a las dificultades propias de la lengua para constituirse como un objeto de conocimiento, principalmente en función de la fijeza o la estabilidad que la enseñanza exige. El argumento sobre el que se sostiene esa resistencia o dificultad es apoyado sobre la postulación (resumida en sus rasgos comunes) de que la lengua es un organismo vivo, imposible de tratar estáticamente, pues ha de ser captado en su real naturaleza y funcionamiento. De este modo, los intentos de fijar la lengua en reglas de funcionamiento o de prescripción serían vanos.

Sin embargo, la pretensión de captar la lengua en su dinamismo y su carácter huidizo tiene, en los textos analizados, más que un fundamento científco, una razón pedagógica, según plantearemos a continuación.

En su reflexión sobre la relación entre la lengua en su totalidad inabarcable y la lengua que puede ser enseñada (representada en esta caso por la gramática), Mibelli admite que

habría que confesarle al que estudia: $1 .^{\circ}$ Como toda ciencia, la Gramática tiene que clasificar sistemáticamente sus 'materiales'. 2. Para tal fin, emplea los casilleros denominados oraciones (suj., pred.). $3 .^{\circ}$ A veces, la unidad lingüística analizable cabe en el casillero. A veces, no; y entonces hay que forzarla (Mibelli 1943, 43, redondas representan cursivas del autor).

Piccardo también señala las insuficiencias de la gramática como retrato del conocimiento de una lengua: "hay en todo idioma una serie de elementos y convenciones imponderables, que no se pueden encerrar en los moldes, siempre limitados, de una preceptiva gramatical" (Piccardo 1956, 94). Rusconi nos indica que "el lenguaje no es un hecho matemático, sino veleidoso como la vida misma, aunque codifique muchos de sus principios" (Rusconi 1956, 13).

Por la manera en que los textos la presentan, la problematización está capturada por las viejas concepciones de gramática como conocimiento sobre la lengua. Por lo tanto, no se cuestionan las posibilidades de que el lenguaje sea descrito científicamente sino de que este sea posible de encasillar en las viejas categorías que imponía la gramática tradicional. Por ejemplo, Da Silva y Guzmán nos dicen, fusionando conocimiento con corrección, que "la corrección gramatical se funda en la conversión, en la regla estable y no en la verdad, ni en la lógica" (Da Silva y Guzmán 1949, 82-83); y Olano, en cambio, muestra esta cuestión como aún abierta y más emparentada con el estudio del lenguaje más allá de las reglas de corrección: "no se pretenderá, sin embargo, expulsar a la lógica del terreno del lenguaje, pero, su preponderancia en él, ha motivado frecuentes errores que, aún en nuestros días, superviven" (Olano 1959, 120).

Estas dificultades para la constitución de un objeto de estudio, cuando se piensa en construir un objeto de enseñanza, son enfrentadas, en la mayoría de los textos, recurriendo a la noción de lengua viva ${ }^{18}$. Da Silva y Guzmán oponen esa noción a la de gramática, diciendo que "la gramática sería la forma reguladora por instantes y el idioma el contenido de la viva idea viva" (Da Silva y Guzmán 1949, 13). Persiste esa idea, dos décadas después, 
cuando Balsas afirma que el material principal de la clase de lengua es "la lengua viva, palpitante, de uso diario por el alumno u otros hablantes, pero siempre dentro de lo circundante" (Balsas 1969, 26).

Vamos observando así cómo la cuestión epistémica resulta en nuestros textos poco discutida, lateralmente mencionada, mezclada entre el problema del estudio mismo del lenguaje y de la enseñanza concreta de la lengua. Se ven reticencias y resistencias, como la de Jesualdo Sosa, a otorgar lugar a "toda especulación puramente teórica y libresca" (Sosa 1955, 9) o aquellas que derivan de todas las cuestiones simultáneas que se tratan en nuestros textos: el saber del hablante y su validez, el lugar de la gramática como corpus de descripciones y de prescripciones tradicionales y el giro pedagógico que se pretende dar. Esto constituye obstáculos o impedimentos para afrontar el problema del saber/conocimiento de lengua puesto en juego en la enseñanza.

En función de la pretendida postura pedagógica emerge una razón instrumental que hace desatender el problema epistémico en la enseñanza de la lengua: la lengua se concibe como instrumento o se limita el lenguaje a la expresión. Esto es evidente en el texto de Jesualdo Sosa (1955), en que todo lo esencial a la enseñanza de la lengua se reduce al lenguaje como medio de expresión y al trabajo pedagógico que el maestro lleva adelante procurando que el alumno alcance recursos para poner sus pensamientos de manifiesto.

Aquello que vemos como renuncia y delegamiento epistémicos se manifiesta al encomendar la tarea del estudio del lenguaje a las disciplinas que, moderna o tradicionalmente, se ocupan de cuestiones de lenguaje: psicología, retórica, gramática, lingüística, etcétera. Esto significa que en gran parte de los textos se ve una desvinculación o desentendimiento de la dimensión epistémica del lenguaje por no considerar que ese debate sea esencial a las discusiones didácticas y pedagógicas pues se piensa que el tratamiento de esas cuestiones es provisto por otros campos del conocimiento y de la actividad de investigación. Da Silva y Guzmán hacen patente esto al afirmar que "las metodologías de la enseñanza del Idioma Español ${ }^{19}$ han cometido y cometen en la actualidad cualquier número de atropellos fisiológicos, (p) sicológicos, biológicos y pedagógicos" (Da Silva y Guzmán 1949, 28) y que "los que elaboran métodos olvidan las nuevas directivas de las recientes concepciones lenguáticas. La Filosofía del Lenguaje, La Estilística, La Lingüística, La Filología han dado nuevos frutos, que debemos aplicar" (Da Silva y Guzmán 1949, 55). Solari también, aunque sin mencionar disciplinas, explica cómo se confía a la ciencia la captura epistémica, tanto del alumno como del conocimiento a enseñar: "la ciencia procuraría establecer las condiciones del proceso y crearía normas adecuadas para lograr frente al niño resultados positivos, de acuerdo con los ideales o fines de la enseñanza" (Solari 1958, 5).

De otro modo, Piccardo (1956), Rusconi (1956) y Olano (1959) muestran fuertes afectaciones en sus maneras de definir los conceptos fundamentales provenientes de la psicología hasta la lingüística (más claramente manifiesto en Olano). En el caso de Rona (1963), la afectación es total o se trata, más bien, de un texto escrito desde la lingüística que se plantea problemas relativos a la enseñanza.

La indeterminación conceptual se ve manifiesta en que no se hallan en los textos distinciones entre lenguaje, lengua, idioma español, idioma
19. Se sumaría aquí la distancia entre la lengua propiamente dicha y el Idioma Español como espacio curricular. 
americano, uruguayo, lengua materna o, incluso, expresión. El punto central de esas indistinciones se observa en que la mayoría de los textos hablan de lenguaje mientras lo que está en cuestión es la enseñanza de la lengua española como lengua correspondiente a la escuela uruguaya por su función de reafirmación nacional.

A los efectos de estos reconocimientos conceptuales, el texto de José Pedro Rona (1963) constituye una excepción pues, al explicar que lo que llama dialectología puede contribuir con lo que él denomina enseñanza de la lengua materna, insiste sobre la pertinencia de las informaciones provistas por las investigaciones lingüísticas para que en la América Hispánica pueda haber una enseñanza de la lengua acorde con la multiplicidad de normas regionales. Remarca, de este modo, el divorcio entre las tradiciones didácticas escolares y la lengua efectivamente presente en las comunidades lingüísticas locales.

Fiorentino (1969), en cambio, aferrada a esas tradiciones que Rona intenta rebatir, pierde la oportunidad de realizar distinciones de este tipo, en tanto dedica su texto a no romper la monolítica asociación entre el español y lengua nacional y escolar uruguaya, ante el desafío planteado por el posible reconocimiento y utilización del portugués como recurso para la alfabetización de algunos grupos de alumnos dentro del país ${ }^{20}$.

Como última limitación u obstáculo impuesto por la orientación pedagógica colocamos lo que llamaríamos enfoque psicológico. Este lleva a que, en la reducción de la lengua/lenguaje a un objeto de aprendizaje, se omita toda preocupación sobre las posibles formas de saber y conocer la lengua en el sentido de su postulación como objeto de estudio y nos queda lo exclusivamente atinente a la incorporación de un objeto ya elaborado, prefijado, acabado, al funcionamiento psicológico del alumno. Como ya indicáramos, se trata de una visión del lenguaje afín a la psicología de las primeras décadas del siglo XX. Un ejemplo de este enfoque aparece en el caso de Piccardo (1956), donde se nota una preocupación por el aprendizaje, en la medida en que se piensa que las diferentes maneras de enfocar la lengua deben concurrir a un desempeño lingüístico mejorado en el alumno. Como determinados por la misma preocupación podemos mencionar a Da Silva y Guzmán (1949), Sosa (1955), Rusconi (1956), Solari (1958), Olano (1959), Rona (1963) y Mieres (1964), dado que todos ellos trabajan sobre los mejores modos de conseguir en el alumno desempeños lingüísticos, que podríamos atribuir a esas imprecisas categorías de tipo psicológico.

\section{Conclusiones}

Estimamos haber logrado, si bien sucintamente, una presentación general de una línea de investigación con trayectoria y con perspectivas de desarrollo, a la vez que una lectura en base a ciertos presupuestos teóricos que permite observar un período de la historia de la escolarización y del pensamiento sobre la enseñanza de la lengua en Uruguay.

Si bien no era el objetivo fundamental de este artículo, optamos por dejar algunas resumidas referencias sobre algunos aspectos históricos, con menciones a instituciones y figuras importantes, tomando los antecedentes al período en el que aquí nos concentramos de modo de visualizar la escena académica uruguaya, en la que situamos los doce textos tomados en cuenta en este análisis.
20. Se trata del momento en que se discutía el proyecto presentado entre 1967 y 1968 por la Dra. Eloísa García Etchegoyen de Lorenzo para alfabetizar en portugués a los niños lusohablantes del norte del Uruguay. Algunos de los aspectos políticos y discursivos de esta propuesta aparecen tratados en Fustes (2016). 
Intentamos tocar especialmente los rasgos pedagógicos en el pensamiento sobre la enseñanza de la lengua y procuramos hacer algunas indicaciones sobre el problema de la dimensión epistémica de la enseñanza.

La época analizada demuestra un andar bastante errático en lo que respecta a la concepción del lenguaje y/o de las lenguas como objeto de estudio. Todo conocimiento elaborado a partir del lenguaje, tanto provenga este de los tradicionales estudios gramaticales como de la moderna lingüística, se ve instrumentalizado para los fines pedagógicos perseguidos, a los cuales la enseñanza quedaba supeditada.

Esto nos permite comenzar a ver los recorridos separados y los cruces entre el desarrollo de los estudios lingüísticos en la escena académica uruguaya y las concepciones propias de la enseñanza de la lengua. Si bien estas reclaman sustento siempre en alguna forma de conocimiento, están afectadas por otros factores propios de lo institucional y lo escolar que en este caso vimos evidenciado en lo que llamamos enfoque pedagógico. 


\section{Q Bibliografía}

" Agustín de Hippona. 1873. “De Magistro”. En CEuvres complètes de Saint Augustin Évêque d'Hippone, tome III, 254-291. Paris: Librairie de Louis Vivès.

" Araújo Villagrán, Horacio. 1920. Los italianos en el Uruguay (diccionario biográfico). Buenos Aires: Escardó \& Araújo Editores.

"Balsas, Héctor. 1969. "El maestro frente a la enseñanza de la lengua”. Revista de la Educación del Pueblo 2.5: 25-28.

" Barrios, Graciela, Luis E. Behares, Adolfo Elizaincín, Beatriz Gabbiani y Susana Mazzolini. 1993. "Planificación y políticas lingüísticas en Uruguay”. Iztapalapa 29: 177-190.

" Barrios, Graciela. 2009. “Incidencia de la estandarización lingüística y de la educación en la uniformidad de variedades y comunidades lingüísticas". Revista Digital de Políticas Lingüísticas 1.1: 30-61.

» Barrios, Graciela. 2011. “La regulación política de la diversidad: academias de lenguas y prescripción idiomática”. En El dardo en la Academia: Esencia y vigencia de las Academias de la lengua española, editado por Silvia Senz y Montserrat Alberte, volumen I, 591-619. Barcelona: Melusina.

» Behares, Luis E. 2005. “Didáctica moderna: ¿más o menos preguntas, más o menos respuestas?”. En Enseñanza del saber: Saber de la enseñanza, coordinado por Luis E. Behares y Susana Colombo de Corsaro, 9-15. Montevideo: Facultad de Humanidades y Ciencias de la Educación.

» Behares, Luis E. 2007. “Enseñar en cuerpo y alma”. ETD: Educação Temática Digital 8: 1-21.

"Behares, Luis E. 2008. "De un cuerpo que responda a la palabra: un retorno a la teoría antigua de la enseñanza". En Cuerpo, lenguaje y enseñanza, compilado por Raumar Rodríguez Giménez, 1-21. Montevideo: Universidad de la República.

» Behares, Luis E. 2011. Enseñanza y producción de conocimiento: La noción de enseñanza en las políticas universitarias uruguayas. Montevideo: CSIC-UdelaR.

»Behares, Luis E. y Claudia Brovetto. 1994. Lo oral y lo escrito en la sociedad uruguaya. Montevideo: Ediciones de la Banda Oriental.

》Behares, Luis E., Eloísa Bordoli, Ana María Fernández Caraballo y Ofelia Ros. 2004. Didáctica mínima: Los acontecimientos del saber. Montevideo: Psicolibros Waslala.

» Berro García, Adolfo, Carlos Martínez Vigil, Víctor Pérez Petit, Alberto Rusconi, José G. Antuña, J. del Rey, E. Laferrière y José Pereira Rodríguez. 1941. “La enseñanza del español en los liceos de la República". Boletín de Filología (Instituto de Estudios Superiores, Montevideo) 3.16/17: 282-287.

" Berro García, Adolfo. 1952. Actividades desarrolladas por el Prof. Adolfo Berro García: Currículum vitae (1906-1952). Montevideo: Monteverde.

» Bordoli, Eloísa. 2011. "Reflexiones en torno a la enseñanza: Notas mínimas”. Didáskomai 2: 93-105.

» Claparède, Edouard. 1933. La psicología y la nueva educación. Madrid: Publicaciones de la Revista de Pedagogía.

" Coll, Magdalena. 1994. “La Banda Oriental en el siglo XVIII: usos, desarrollo y difusión de la lengua escrita”. Anuario de Lingüística Hispánica 10: 25-37. 
»Comenius, Jan Amós. 1986 [1632]. Didactica magna. Madrid: Akal.

»Coseriu, Eugenio. 1952. Sistema, norma y habla. Montevideo: Facultad de Humanidades y Ciencias.

»Coseriu, Eugenio. 1958. Sincronía, diacronía e historia. Montevideo: Facultad de Humanidades y Ciencias.

»Crisipo de Solos. 2008. Lógica estoica. Traducción española de Adrián Castillo, según edición de Johannes von Arnim. Montevideo: Facultad de Humanidades y Ciencias de la Educación.

»Da Silva y Silvera, Andrés y M. E. Guzmán de Da Silva. 1949. Poemario metodológico de la lengua española. Montevideo: Ciudadela.

»Eco, Umberto. 1973. Segno. Milano: Istituto Editoriale Internazionale.

» Fernández, Ana María. 2006. “'Lo niño’ y el psicoanálisis: ¿posibilidad o imposibilidad? ETD: Educação Temática Digital 8: 20-48.

» Fernández, Ana María. 2008. “Del amur y la verité en la enseñanza”. Tiresias 2: 95-110.

» Fernández, Ana María. 2010. “Del matema (mathémata-mathésis) y la transmisión”. Fermentario 4, <http://www.fermentario.fhuce.edu.uy/index.php/fermentario/article/ view/32>.

» Fustes, Juan Manuel. 2010. “De Saussure ante los lingüistas del siglo XIX y el problema del sujeto”. Cadernos de Estudos Lingüísticos 52.1: 55-64.

» Fustes, Juan Manuel. 2014. “La condición de hablante del enseñante de lengua extranjera, entre la enseñanza y la transmisión”. Didáskomai 5: 103-113.

» Fustes, Juan Manuel. 2016. “Las concepciones sobre la enseñanza de la lengua en la escena académica uruguaya entre 1920 y 1970". Tesis de maestría, Universidad de la República.

"Henry, Paul. 1992. A ferramenta imperfeita: Língua, sujeito e discurso. Campinas: Editora da UniCamp.

» Lacan, Jacques. 1974-1975. Seminario 22 - R.S.I. Buenos Aires: Escuela Freudiana de Buenos Aires.

» Lacan, Jacques. 1982. “'Le Symbolique, l'imaginaire et le Réel', conférence à la Société française de psychanalyse (8-7-1953)". Bulletin de l'Association Freudienne 1: 4-13.

»Lacan, Jacques. 2005 [1966]. “Función y campo de la palabra y del lenguaje en psicoanálisis”. En Escritos I, 227-310. Buenos Aires: Siglo Veintiuno Editores Argentina.

»Lemos, Cláudia T. G. de. 1995. “Língua e discurso na na teorização sobre aquisição de linguagem”. Letras de Hoje 30.4: 9-28.

»Lemos, Cláudia. 200o. "Questioning the notion of development: The case of language acquisition”. Culture and Psychology 6: 169-181.

» Luisi, Luisa. 1924. “Prólogo”. En Cervantes: Libro de lenguaje, Roberto Abadie y Humberto Zarrilli, 5-7. Montevideo: Imprenta Latina.

» Melo, Maria de Fátima Vilar de y Glória Maria Monteiro de Carvalho. 2014. “Pontos polêmicos na leitura que Lacan fez de Saussure”. Matraga 21.34: 168-179.

» Mibelli, Américo. 1943. Lenguaje: palabra, gesto en la enseñanza media uruguaya. Montevideo: Editorial Independencia.

» Mieres, Celia. 1964. "Cursos de idioma español en los liceos”. Anales del Instituto de Profesores “Artigas" 9: 70-106. 
》 Milner, Jean-Claude. 1978. L'amour de la langue. Paris: Seuil.

» Milner, Jean-Claude. 1989. Introduction à une science du langage. Paris: Seuil.

» Muresanu, Constantin. 1930. L'éducation de l'adolescent par la composition libre. Paris: Delachaux \& Niestlé S. A.

» Olano Pagola, Floro. 1959. “Adquisición del signo”. Boletín de Filología 8.55/56/57: 107-142.

» Oroño, Mariela. 2010. “El lugar de la lengua en la configuración de la patria: el plan del libro cuarto de lectura de Abadie y Zarrilli (Uruguay, 1941)”, Revista Digital de Políticas Lingüísticas 2: 20-39.

"Oroño, Mariela. 2011. "La escuela pública uruguaya y el idioma nacional en dos períodos históricos: fines del siglo XIX y mediados del siglo XX”. Letras 21.42: 217-247.

»Oroño, Mariela. 2014. “La escuela y la lengua en la construcción de la identidad nacional uruguaya: los libros de lectura usados en la escuela pública en los años 40 del siglo XX”. Boletín de Filología 49.2: 215-236.

» Oroño, Mariela. 2016a. "La escuela en la construcción de las fronteras culturales y lingüísticas en el Uruguay de fines del siglo XIX”. Páginas de Educación 9.1: 137-167.

» Oroño, Mariela. 2016b. “La polémica sobre acentuación ortográfica entre Carlos Martínez Vigil y Fidelis del Solar”. Nueva Revista del Pacífico 64: 67-96.

" Pardo, Vincenzo. 2011. “Aristote, Saussure et la convention du signe”. Studii de Știință și Cultură 7.1.24: 19-34.

»Perea y Alonso, Sixto. 1936. “Notas sobre nomenclatura en las ciencias del lenguaje”, Boletín de Filología, Tomo I, 1: 17-22.

»Pereira Rodríguez, José. 1940. Enseñanza de la redacción y composición: teoría y práctica. Buenos Aires: Kapelusz.

» Piccardo, Luis Juan. 1956. “Gramática y enseñanza”. Anales del Instituto de Profesores “Artigas" 1: 31-52.

»Rona, José Pedro. 1963. "Relación entre la investigación dialectológica y la enseñanza de la lengua materna”. Revista de Lingüística Aplicada 2: 1-12.

»Rusconi, Alberto. 1956. Metodología de la enseñanza de la lengua española y la literatura en secundaria. Montevideo: s/e.

» Solari, Héctor Enrique. 1958. “Hablar, leer, escribir”. Anales 2.21.10/11/12: 5-56.

» Sosa, Jesualdo. 1955. “Metodología de la expresión infantil”. Anales de Instrucción Primaria 2.18.10/11/12: 5-28.

"Suenaga, Akatane. 1999. "Des deux arbitraires, absolu et relatif, à un arbitraire "Primaire» - le fait linguistique et le devenir du signe chez Saussure”. Cahiers Ferdinand de Saussure 52: 189-200.

» Todorov, Tzvetan. 1977. Théories du symbole. Paris: Seuil.

»Venturini, Joaquín. 2012. "La cuestión del don y sus derivas para indagar en la no equivalencia entre enseñanza y aprendizaje”. Didáskomai 3: 67-80.

»Venturini, Joaquín. 2015. “Derivas de Herbart e Itard en el siglo XX: Apuntes para una genealogía de la Escuela Nueva”. Didáskomai 6: 147-173. 